\title{
INTUITIONISTIC FUZZY PRIORITIZED OPERATORS AND THEIR APPLICATION IN MULTI-CRITERIA GROUP DECISION MAKING
}

\author{
Dejian YU \\ School of Information, Zhejiang University of Finance and Economics, \\ Hangzhou 310018, China
}

Received 15 June 2011; accepted 15 November 2011

\begin{abstract}
Intuitionistic fuzzy set is a very useful tool to depict uncertainty. Lots of multi-criteria group decision making methods under intuitionistic fuzzy environment have been developed. Current methods are under the assumption that the criteria and the decision makers are at the same priority level. However, in real group decision making problems, criteria and decision makers have different priority level commonly. In this paper, multi-criteria group decision making problems where there exists a prioritization relationship over the criteria and decision makers are studied. First, the intuitionistic fuzzy prioritized weighted average (IFPWA) and the intuitionistic fuzzy prioritized weighted geometric (IFPWG) operators are proposed. Then, some of their desirable properties are investigated in detail. Furthermore, the procedure of multi-criteria group decision making based on the proposed operators is given under intuitionistic fuzzy environment. Finally, a practical example about talent introduction is provided to illustrate the developed method.
\end{abstract}

Keywords: Multi-criteria group decision making, aggregation operator, intuitionistic fuzzy set, intuitionistic fuzzy prioritized weighted average (IFPWA) operator, intuitionistic fuzzy prioritized geometric (IFPWG) operator.

Reference to this paper should be made as follows: Yu, D. 2013. Intuitionistic fuzzy prioritized operators and their application in multi-criteria group decision making, Technological and Economic Development of Economy 19(1): 1-21.

JEL Classification: C43, D81.

\section{Introduction}

Atanasov (1986) extended the concept of fuzzy sets which was proposed by Zadeh (1965), and introduced the intuitionistic fuzzy set (IFS). IFS is characterized by a membership degree and a non-membership degree, so it is more effective to deal with uncertainty and vagueness in real applications than Zadeh's fuzzy sets. Since its appearance, IFS has been investigated by

Corresponding address:

D. Yu E-mail: yudejian62@126.com 
many researchers and applied to many fields, such as pattern recognition (Hong, Choi 2000; Hung, Yang 2004, 2007), market prediction (Liang, Shi 2003) and decision making (Liu 2009; Liu, Wang 2007; Chen 2011; Li 2010; Wei 2010a, b; Wei 2011; Wei et al. 2011a, b; Wei et al. 2012; Wei, Zhao 2012; Yu 2012; Yu 2013; Yu et al. 2012; Xia et al. 2012).

As a hot topic in the theory of IFS, intuitionistic fuzzy information aggregation has been investigated widely by many researchers from different points of view. According to the relationships between the aggregated arguments, the aggregation operators can be divided into two categories. The first category is based on the assumption that the aggregated arguments are independent. Based on the OWA operator (Yager 1988), Xu (2007) developed some aggregation operators, such as the intuitionistic fuzzy weighted averaging (IFWA) operator, the intuitionistic fuzzy ordered weighted averaging (IFOWA) operator, the intuitionistic hybrid aggregation (IFHA) operator. Xu and Yager (2006) developed some geometric aggregation operators for intuitionistic fuzzy values (IFVs), such as the intuitionistic fuzzy weighted geometric (IFWG) operator, the intuitionistic fuzzy ordered weighted geometric (IFOWG) operator, and the intuitionistic fuzzy hybrid geometric (IFHG) operator. Based on the GOWA operator (Yager 2004a), Zhao et al. (2010) developed some generalized aggregation operators, such as the generalized intuitionistic fuzzy weighted averaging (GIFWA) operator, the generalized intuitionistic fuzzy ordered weighted averaging (GIFOWA) operator, and the generalized intuitionistic fuzzy hybrid averaging (GIFHA) operator. In the second category it is assumed that there are interactions phenomena among the decision making criteria. To deal with this issue, Xu (2011a) extended the power average (Yager 2001) to intuitionistic fuzzy environment and introduced a series of operators for aggregating intuitionistic fuzzy values (IFVs) whose weighting vectors depend on the input arguments. Xu (2011b) applied the Bonferroni mean (BM) (Yager 2009a) to intuitionistic fuzzy environment and introduced the intuitionistic fuzzy Bonferroni mean (IFBM) and the weighted Bonferroni mean (WIFBM) whose characteristic is that they can not only consider the importance of each criterion but also reflect the interrelationship of the individual criteria. Motivated by the induced Choquet ordered averaging operator (Yager 2004b), Xu (2010), Tan and Chen (2010) and Tan (2011) developed some intuitionistic fuzzy correlative operators, such as the intuitionistic fuzzy Choquet average (IFCA) operator, and the intuitionistic fuzzy Choquet geometric (IFCG) operator.

The above aggregation operators for IFVs is assuming that the criteria are at the same priority level although some of them consider the correlation phenomena between criteria. They are characterized by the ability to trade-off between criteria. For example, if $C_{i}$ and $C_{j}$ are two criteria with the weight $\omega_{i}$ and $\omega_{j}$ respectively. By the above aggregation operator, we can compensate for a decrease of $\theta$ in satisfaction to criteria $C_{i}$ by gain $\frac{\omega_{j}}{\omega_{i}} \theta$ in satisfaction to criteria $C_{j}$. However, in many real decision making problems, this kind of compensation between criteria is not feasible. Consider the situation in which a woman is making a decision based on consideration of powdered milk cost and safety for her child. 
She should not allow a benefit with respect to cost of powdered milk compensate for a loss in safety. This is a typical kind of prioritization of the criteria, i.e. Safety has a higher priority than cost. When making decisions in Chinese universities, criteria desired by president usually have a higher priority than professor. Yager (2008) first investigated this kind of problem by introducing the prioritized "and" and "or" operators. Then, Yager (2009b) and Yager et al. (2011) have paid attention on this issue. In this paper, we research the aggregation method for IFVs which has prioritization relationships between the criteria. To do this, the remainder of this paper is organized as follows. In Section 1, we briefly review some basic concepts. Section 2 proposes the intuitionistic fuzzy prioritized weighted average (IFPWA) operator and intuitionistic fuzzy prioritized geometric average (IFPWG) operator to aggregate the IFVs, whose desirable properties are also studied in this Section. In Section 3, we develop a method for multi-criteria group decision making based on the proposed operators under intuitionistic fuzzy environment. A practical example about the introduction of talents is provided in Section 4.

\section{Basic concepts and operations}

Definition 1 (Atanassov 1986). Let $X=\left\{x_{1}, x_{2}, \cdots, x_{n}\right\}$ be fixed. An inuitionistic fuzzy set (IFS) $A$ on $X$ can be defined as:

$$
A=\left\{\left(x_{i}, \mu_{A}\left(x_{i}\right), v_{A}\left(x_{i}\right)\right) \mid x_{i} \in X\right\},
$$

where the functions $\mu_{A}\left(x_{i}\right)$ and $v_{A}\left(x_{i}\right)$ denote the membership and non-membership of $x_{i}$ to $X$ in $A$ with the condition that

$$
0 \leq \mu_{A}\left(x_{i}\right) \leq 1,0 \leq v_{A}\left(x_{i}\right) \leq 1, \mu_{A}\left(x_{i}\right)+v_{A}\left(x_{i}\right) \leq 1,
$$

and $\pi_{A}\left(x_{i}\right)=1-\mu_{A}\left(x_{i}\right)-v_{A}\left(x_{i}\right)$ is called a hesitation degree of $x_{i}$ to $A$.

$\mathrm{Xu}$ (2007) named the pair $\left(\mu_{\alpha}, v_{\alpha}\right)$ intuitionistic fuzzy value (IFV) denoted as $\alpha$ with the condition $0 \leq \mu_{\alpha}, v_{\alpha} \leq 1, \mu_{\alpha}+v_{\alpha} \leq 1$. Chen and Tan (1994) introduced the score function $s(\alpha)=t_{\alpha}-f_{\alpha}$ to get the score of $\alpha$, Then, Hong and Choi (2000) defined an accuracy function $H(\alpha)=\mu_{\alpha}+v_{\alpha}$ to evaluate the accuracy degree of $\alpha$. Xu and Yager (2006) gave an total order relation between two IFVs $\alpha$ and $\beta$.

If $s(\alpha)<s(\beta)$, then $\alpha<\beta$;

If $s(\alpha)=s(\beta)$, then

i) If $h(\alpha)=h(\beta)$, then $\alpha=\beta$;

ii) If $h(\alpha)<h(\beta)$, then $\alpha<\beta$.

It should be noted that the score function $s(\alpha)$ is between -1 and 1 . In order to facilitate the following study, we introduce another score function proposed by Liu (2005). She introduced a score function $S(\alpha)=\frac{1+\mu_{\alpha}-v_{\alpha}}{2}$ to get the score of $\alpha$. Since $0 \leq \mu_{\alpha}, v_{\alpha} \leq 1$, $\mu_{\alpha}+v_{\alpha} \leq 1$, we can easily get $0 \leq S(\alpha) \leq 1$. When comparing Chen's and Liu's score func- 
tions, it can be concluded that, if $s(\alpha)<s(\beta)(s(\alpha) \geq s(\beta))$, then $S(\alpha)<S(\beta)(S(\alpha) \geq S(\beta))$; on the other hand, if $S(\alpha)<S(\beta)(S(\alpha) \geq S(\beta))$, then $s(\alpha)<s(\beta)((s(\alpha) \geq s(\beta))$. Therefore, if we replace the Chen's score function by Liu's score function, the order relation between two IFVs $\alpha$ and $\beta$ introduced by Xu and Yager (2006) are also valid.

Let us denote by $L$ the lattice of non-empty IFVs $L=\left\{(a, b) \mid(a, b) \in[0,1]^{2}, a+b \leq 1\right\}$ with the partial order $\leq_{L}$ defined as $(a, b) \leq_{L}(c, d) \Leftrightarrow a \leq c \& b \geq d$. The top and bottom elements are $1_{L}=(1,0)$ and $0_{L}=(0,1)$ respectively. (Beliakov et al. 2011; Deschrijver, Kerre 2003). Let $V$ be the set of all intuitionistic fuzzy values (IFVs) with $\leq_{L}$.

Remark 1: If two IFVs $\alpha \leq_{L} \beta$, then we have, $\alpha \leq \beta$.

For three IFVs $\alpha, \alpha_{1}, \alpha_{2} \in V$, some operational laws were given as follows (Xu, Yager 2006).

1) $\alpha_{1} \oplus \alpha_{2}=\left(\mu_{\alpha_{1}}+\mu_{\alpha_{2}}-\mu_{\alpha_{1}} \mu_{\alpha_{2}}, v_{\alpha_{1}} v_{\alpha_{2}}\right)$;

2) $\alpha_{1} \otimes \alpha_{2}=\left(\mu_{\alpha_{1}} \mu_{\alpha_{2}}, v_{\alpha_{1}}+v_{\alpha_{2}}-v_{\alpha_{1}} v_{\alpha_{2}}\right)$;

3) $\lambda \alpha=\left(1-\left(1-\mu_{\alpha}\right)^{\lambda}, v_{\alpha}{ }^{\lambda}\right), \lambda>0$;

4) $\alpha^{\lambda}=\left(\mu_{\alpha}^{\lambda}, 1-\left(1-v_{\alpha}\right)^{\lambda}\right), \lambda>0$.

The Prioritized Average (PA) operator was originally introduced by Yager (2008), which was defined as follows:

Definition 2 (Yager 2008). Let $C=\left\{C_{1}, C_{2}, \ldots, C_{n}\right\}$ be a collection of criteria and there is a prioritization between the criteria expressed by the linear ordering $C_{1} \succ C_{2} \succ C_{3} \cdots \succ C_{n}$, indicate criteria $C_{j}$ has a higher priority than $C_{k}$ if $j<k$. The value $C_{j}(x)$ is the performance of any alternative $x$ under criteria $C_{j}$, and satisfies $C_{j}(x) \in[0,1]$. If

$$
P A\left(C_{i}(x)\right)=\sum_{j=1}^{n} w_{j} C_{j}(x)
$$

where $w_{j}=\frac{T_{j}}{\sum_{j=1}^{n} T_{j}}, T_{j}=\prod_{k=1}^{j-1} C_{k}(x)(j=2, \ldots, n), T_{1}=1$. Then PA is called the prioritized average (PA) operator.

\section{Intuitionistic fuzzy prioritized average operators}

In this Section, we shall investigate the PA operator under intuitionistic fuzzy environments. Based on Definition 2, we give the definition of the IFPWA as follows:

Definition 3. Let $\alpha_{j}=\left(\mu_{\alpha_{j}}, v_{\alpha_{j}}\right)(j=1,2, \ldots, n)$ be a collection of IFVs, and let IFPWA: $V^{n} \rightarrow V$, if

$$
\operatorname{IFPWA}\left(\alpha_{1}, \alpha_{2}, \ldots, \alpha_{n}\right)=\frac{T_{1}}{\sum_{j=1}^{n} T_{j}} \alpha_{1} \oplus \frac{T_{2}}{\sum_{j=1}^{n} T_{j}} \alpha_{2} \oplus \cdots \oplus \frac{T_{n}}{\sum_{j=1}^{n} T_{j}} \alpha_{n},
$$

then the function IFPWA is called an intuitionistic fuzzy prioritized weighted average (IFPWA) operator, where $T_{j}=\prod_{k=1}^{j-1} S\left(\alpha_{k}\right) \quad(j=2, \ldots, n), T_{1}=1$ and $S\left(\alpha_{k}\right)$ is the score of IFV $\alpha_{k}$. 
Based on operations of the IFVs described in Section 1, we can drive the Theorem 1.

Theorem 1. Let $\alpha_{j}=\left(\mu_{\alpha_{j}}, v_{\alpha_{j}}\right)(j=1,2, \ldots, n)$ be a collection of IFVs, then their aggregated value by using the IFPWA operator is also an IFV, and

$$
\operatorname{IFPWA}\left(\alpha_{1}, \alpha_{2}, \ldots, \alpha_{n}\right)=\left(1-\prod_{j=1}^{n}\left(1-\mu_{\alpha_{j}}\right)^{\frac{T_{j}}{\sum_{j=1}^{n} T_{j}}}, \prod_{j=1}^{n}\left(v_{\alpha_{j}}\right)^{\frac{T_{j}}{\sum_{j=1}^{n} T_{j}}}\right),
$$

where $T_{j}=\prod_{k=1}^{j-1} S\left(\alpha_{k}\right) \quad(j=2, \ldots, n), T_{1}=1$ and $S\left(\alpha_{k}\right)$ is the score of IFV $\alpha_{k}$.

Proof. The first result follows quickly from Definition 1 and Theorem 1 . In the following, we prove

$$
\begin{aligned}
& \operatorname{IFPWA}\left(\alpha_{1}, \alpha_{2}, \ldots, \alpha_{n}\right)=\frac{T_{1}}{\sum_{j=1}^{n} T_{j}} \alpha_{1} \oplus \frac{T_{2}}{\sum_{j=1}^{n} T_{j}} \alpha_{2} \oplus \cdots \oplus \frac{T_{n}}{\sum_{j=1}^{n} T_{j}} \alpha_{n}= \\
& \left(1-\prod_{j=1}^{n}\left(1-\mu_{\alpha_{j}}\right) \frac{T_{j}}{\sum_{j=1}^{n} T_{j}}, \prod_{j=1}^{n}\left(v_{\alpha_{j}}\right) \frac{T_{j}}{\sum_{j=1}^{n} T_{j}}\right) \text {. }
\end{aligned}
$$

By using mathematical induction on $n$ :

1) For $n=2$ : Since

$$
\begin{aligned}
& \frac{T_{1}}{\sum_{j=1}^{n} T_{j}} \alpha_{1}=\left(1-\left(1-\mu_{\alpha_{1}}\right) \frac{T_{1}}{\sum_{j=1}^{n} T_{j}},\left(v_{\alpha_{1}}\right) \frac{T_{1}}{\sum_{j=1}^{n} T_{j}}\right) ; \\
& \frac{T_{2}}{\sum_{j=1}^{n} T_{j}} \alpha_{2}=\left(1-\left(1-\mu_{\alpha_{2}}\right) \frac{T_{2}}{\sum_{j=1}^{n} T_{j}},\left(v_{\alpha_{2}}\right) \frac{T_{2}}{\sum_{j=1}^{n} T_{j}}\right) .
\end{aligned}
$$

We have

$$
\begin{gathered}
\operatorname{IFPWA}\left(\alpha_{1}, \alpha_{2}\right)=\frac{T_{1}}{\sum_{j=1}^{n} T_{j}} \alpha_{1} \oplus \frac{T_{2}}{\sum_{j=1}^{n} T_{j}} \alpha_{2}= \\
\left(1-\left(1-\mu_{\alpha_{1}}\right) \frac{T_{1}}{\sum_{j=1}^{n} T_{j}}\left(1-\mu_{\alpha_{2}}\right) \frac{T_{2}}{\sum_{j=1}^{n} T_{j}},\left(v_{\alpha_{1}}\right) \frac{T_{1}}{\sum_{j=1}^{n} T_{j}}\left(v_{\alpha_{2}}\right) \frac{T_{2}}{\sum_{j=1}^{n} T_{j}}\right) .
\end{gathered}
$$

2) If Eq. (6) holds for $n=k$, that is

$$
\begin{gathered}
\operatorname{IFPWA}\left(\alpha_{1}, \alpha_{2}, \ldots, \alpha_{k}\right)=\frac{T_{1}}{\sum_{j=1}^{n} T_{j}} \alpha_{1} \oplus \frac{T_{2}}{\sum_{j=1}^{n} T_{j}} \alpha_{2} \oplus, \ldots, \frac{T_{k}}{\sum_{j=1}^{n} T_{j}} \alpha_{k}= \\
\left(1-\prod_{j=1}^{k}\left(1-\mu_{\alpha_{j}}\right) \frac{T_{j}}{\sum_{j=1}^{n} T_{j}}, \prod_{j=1}^{k}\left(v_{\alpha_{j}}\right) \frac{T_{j}}{\sum_{j=1}^{n} T_{j}}\right),
\end{gathered}
$$


then, when $n=k+1$, by the operational laws described in Section 1, we have

$$
\begin{gathered}
\operatorname{IFPWA}\left(\alpha_{1}, \alpha_{2}, \ldots, \alpha_{k+1}\right)= \\
\left(1-\prod_{j=1}^{k}\left(1-\mu_{\alpha_{j}}\right) \frac{T_{j}}{\sum_{j=1}^{n} T_{j}}, \prod_{j=1}^{k}\left(v_{\alpha_{j}}\right) \frac{T_{j}}{\sum_{j=1}^{n} T_{j}}\right) \oplus\left(1-\left(1-\mu_{\alpha_{k+1}}\right) \frac{T_{k+1}}{\sum_{j=1}^{n} T_{j}},\left(v_{\alpha_{k+1}}\right) \frac{T_{k+1}}{\sum_{j=1}^{n} T_{j}}\right)= \\
\left(1-\prod_{j=1}^{k+1}\left(1-\mu_{\alpha_{j}}\right) \frac{T_{j}}{\sum_{j=1}^{n} T_{j}}, \prod_{j=1}^{k+1}\left(v_{\alpha_{j}}\right) \frac{T_{j}}{\sum_{j=1}^{n} T_{j}}\right),
\end{gathered}
$$

i.e. Eq. (6) holds for $n=k+1$. Thus, Eq. (6) holds for all $n$. Then

$$
\operatorname{IFPWA}\left(\alpha_{1}, \alpha_{2}, \ldots, \alpha_{n}\right)=\left(1-\prod_{j=1}^{n}\left(1-\mu_{\alpha_{j}}\right)^{\frac{T_{j}}{\sum_{j=1}^{n} T_{j}}}, \prod_{j=1}^{n}\left(v_{\alpha_{j}}\right)^{\frac{T_{j}}{\sum_{j=1}^{n} T_{j}}}\right) .
$$

Now, we look at some desirable properties of the IFPWA operator.

Theorem 2. (Idempotency) Let $\alpha_{j}=\left(\mu_{\alpha_{j}}, v_{\alpha_{j}}\right)(j=1,2, \ldots, n)$ be a collection of IFVs, where $T_{j}=\prod_{k=1}^{j-1} S\left(\alpha_{k}\right)(j=2, \ldots, n), T_{1}=1$ and $S\left(\alpha_{k}\right)$ is the score of IFV $\alpha_{k}$. If all $\alpha_{j}(j=1,2, \ldots, n)$ are equal, i.e. $\alpha_{j}=\alpha$, for all $j$, then

$$
\operatorname{IFPWA}\left(\alpha_{1}, \alpha_{2}, \ldots, \alpha_{n}\right)=\alpha .
$$

Proof. By Definition 3, we have

$$
\begin{gathered}
\operatorname{IFPWA}\left(\alpha_{1}, \alpha_{2}, \ldots, \alpha_{n}\right)= \\
\frac{T_{1}}{\sum_{j=1}^{n} T_{j}} \alpha_{1} \oplus \frac{T_{2}}{\sum_{j=1}^{n} T_{j}} \alpha_{2} \oplus \cdots \oplus \frac{T_{n}}{\sum_{j=1}^{n} T_{j}} \alpha_{n}= \\
\frac{T_{1}}{\sum_{j=1}^{n} T_{j}} \alpha \oplus \frac{T_{2}}{\sum_{j=1}^{n} T_{j}} \alpha \oplus \cdots \oplus \frac{T_{n}}{\sum_{j=1}^{n} T_{j}} \alpha= \\
\frac{\sum_{j=1}^{n} T_{j}}{\sum_{j=1}^{n} T_{j}} \alpha=\alpha .
\end{gathered}
$$

Corollary 1. If $\alpha_{j}=\left(\mu_{\alpha_{j}}, v_{\alpha_{j}}\right)(j=1,2, \ldots, n)$ is a collection of the largest IFVs, i.e., $\alpha_{j}=\alpha^{*}=(1,0)$, for all $j$, then

$$
\operatorname{IFPWA}\left(\alpha_{1}, \alpha_{2}, \ldots, \alpha_{n}\right)=\left(\alpha^{*}, \alpha^{*}, \ldots, \alpha^{*}\right)=(1,0),
$$

which is also the largest IFV. 
Proof. Similar to the proof of Theorem 2, we can get Corollary easily. then

Corollary 2. (Non-compensatory) If $\alpha_{1}=\left(\mu_{\alpha_{1}}, v_{\alpha_{1}}\right)$ is the smallest IFV, i.e., $\alpha_{1}=\alpha_{*}=(0,1)$,

$$
\operatorname{IFPWA}\left(\alpha_{1}, \alpha_{2}, \ldots, \alpha_{n}\right)=\operatorname{IFPWA}\left(\alpha_{*}, \alpha_{2}, \ldots, \alpha_{n}\right)=(0,1),
$$

which is also the smallest IFV.

Proof. Since $\alpha_{1}=(0,1)$, then by the definition of the score function defined in Section 1 , we have,

$$
S\left(\alpha_{1}\right)=0
$$

Since

$$
T_{j}=\prod_{k=1}^{j-1} S\left(\alpha_{k}\right) \quad(j=2, \ldots, n) \text { and } T_{1}=1
$$

We have

$$
\begin{gathered}
T_{j}=\prod_{k=1}^{j-1} S\left(\alpha_{k}\right)=S\left(\alpha_{1}\right) \times S\left(\alpha_{2}\right) \times \cdots \times S\left(\alpha_{j-1}\right)=0 \times S\left(\alpha_{2}\right) \times \cdots \times S\left(\alpha_{j-1}\right)=0 \quad(j=2, \ldots, n) ;(18 \\
\sum_{j=1}^{n} T_{j}=1
\end{gathered}
$$

By Definition 3, we have

$$
\begin{gathered}
\operatorname{IFPWA}\left(\alpha_{1}, \alpha_{2}, \ldots, \alpha_{n}\right)=\frac{T_{1}}{\sum_{j=1}^{n} T_{j}} \alpha_{1} \oplus \frac{T_{2}}{\sum_{j=1}^{n} T_{j}} \alpha_{2} \oplus \cdots \oplus \frac{T_{n}}{\sum_{j=1}^{n} T_{j}} \alpha_{n}= \\
\frac{1}{1} \alpha_{1} \oplus \frac{0}{1} \alpha_{2} \oplus \cdots \oplus \frac{0}{1} \alpha_{n}=\alpha_{1}=(0,1) .
\end{gathered}
$$

Corollary 2 indicated that when the satisfaction to the criteria which owns the highest priority is the smallest IFV, we can't get any compensation from other criteria even if they are satisfied.

Theorem 3. (Boundary) Let $\alpha_{j}=\left(\mu_{\alpha_{j}}, v_{\alpha_{j}}\right)(j=1,2, \ldots, n)$ be a collection of IFVs, where $T_{j}=\prod_{k=1}^{j-1} S\left(\alpha_{k}\right) \quad(j=2, \ldots, n), T_{1}=1$ and $S\left(\alpha_{k}\right)$ is the score of IFV $\alpha_{k}$, and let

Then

$$
\alpha^{-}=\left(\min _{j}\left(\mu_{\alpha_{j}}\right), \max _{j}\left(v_{\alpha_{j}}\right)\right), \alpha^{+}=\left(\max _{j}\left(\mu_{\alpha_{j}}\right), \min _{j}\left(v_{\alpha_{j}}\right)\right) \text {. }
$$

$$
\alpha^{-} \leq \operatorname{IFPWA}\left(\alpha_{1}, \alpha_{2}, \ldots, \alpha_{n}\right) \leq \alpha^{+} .
$$

Proof. Since $\min _{j}\left(\mu_{\alpha_{j}}\right) \leq \mu_{\alpha_{j}} \leq \max _{j}\left(\mu_{\alpha_{j}}\right)$ and $\min _{j}\left(v_{\alpha_{j}}\right) \leq v_{\alpha_{j}} \leq \max _{j}\left(v_{\alpha_{j}}\right)$, for all $j$, then

$$
\prod_{j=1}^{n}\left(1-\mu_{\alpha_{j}}\right)^{\frac{T_{j}}{\sum_{j=1}^{n} T_{j}}} \geq \prod_{j=1}^{n}\left(1-\max _{j}\left(\mu_{\alpha_{j}}\right)\right)^{\frac{T_{j}}{\sum_{j=1}^{n} T_{j}}}=1-\max _{j}\left(\mu_{\alpha_{j}}\right),
$$

and then

$$
1-\prod_{j=1}^{n}\left(1-\mu_{\alpha_{j}}\right)^{\frac{T_{j}}{\sum_{j=1}^{n} T_{j}}} \leq \max _{j}\left(\mu_{\alpha_{j}}\right) .
$$


Similarly, we have

and

$$
1-\prod_{j=1}^{n}\left(1-\mu_{\alpha_{j}}\right)^{\frac{T_{j}}{\sum_{j=1}^{n} T_{j}}} \geq \min _{j}\left(\mu_{\alpha_{j}}\right)
$$

$$
\prod_{j=1}^{n}\left(\min _{j}\left(v_{\alpha_{j}}\right)\right)^{\frac{T_{j}}{\sum_{j=1}^{n} T_{j}}} \leq \prod_{j=1}^{n}\left(v_{\alpha_{j}}\right)^{\frac{T_{j}}{\sum_{j=1}^{n} T_{j}}} \leq \prod_{j=1}^{n}\left(\max _{j}\left(v_{\alpha_{j}}\right)\right)^{\frac{T_{j}}{\sum_{j=1}^{n} T_{j}}} .
$$

Let IFPWA $\left(\alpha_{1}, \alpha_{2}, \ldots, \alpha_{n}\right)=\alpha=\left(\mu_{\alpha}, v_{\alpha}\right)$, then we have,

$$
\begin{gathered}
\min _{j}\left(\mu_{\alpha_{j}}\right) \leq \mu_{\alpha} \leq \max _{j}\left(\mu_{\alpha_{j}}\right) ; \\
\min _{j}\left(v_{\alpha_{j}}\right) \leq v_{\alpha} \leq \max _{j}\left(v_{\alpha_{j}}\right) .
\end{gathered}
$$

From Eqs. (26) and (27), we can get Eqs. (28) and (29) easily.

Therefore,

$$
\begin{aligned}
& \mu_{\alpha} \geq \min _{j}\left(\mu_{\alpha_{j}}\right), v_{\alpha} \leq \max _{j}\left(v_{\alpha_{j}}\right) ; \\
& \mu_{\alpha} \leq \max _{j}\left(\mu_{\alpha_{j}}\right), v_{\alpha} \geq \min _{j}\left(v_{\alpha_{j}}\right) .
\end{aligned}
$$

$$
\alpha^{-} \leq_{L} \operatorname{IFPWA}\left(\alpha_{1}, \alpha_{2}, \ldots, \alpha_{n}\right) \leq_{L} \alpha^{+} .
$$

Based on remark 1, we have,

$$
\alpha^{-} \leq \operatorname{IFPWA}\left(\alpha_{1}, \alpha_{2}, \ldots, \alpha_{n}\right) \leq \alpha^{+},
$$

which completes the proof of Theorem 3.

Theorem 4. (Monotonicity) Let $\alpha_{j}=\left(\mu_{\alpha_{j}}, v_{\alpha_{j}}\right)$ and $\alpha_{j}^{*}=\left(\mu_{\alpha_{j}^{*}}, v_{\alpha_{j}^{*}}\right) \quad(j=1,2, \ldots, n)$ be two collections of IFVs, $T_{j}=\prod_{k=1}^{j-1} S\left(\alpha_{k}\right), T_{j}^{*}=\prod_{k=1}^{j-1} S\left(\alpha_{k}^{*}\right)(j=2, \ldots, n), T_{1}=T_{1}^{*}=1, S\left(\alpha_{k}\right)$ is the score of IFV $\alpha_{k}, S\left(\alpha_{k}^{*}\right)$ is the score of IFV $\alpha_{k}^{*}$ if $\mu_{\alpha_{j}} \leq \mu_{\alpha_{j}^{*}}$ and $v_{\alpha_{j}} \geq v_{\alpha_{j}^{*}}$, for all $j$, then

$$
\operatorname{IFPWA}\left(\alpha_{1}, \alpha_{2}, \ldots, \alpha_{n}\right) \leq \operatorname{IFPWA}\left(\alpha_{1}^{*}, \alpha_{2}^{*}, \ldots, \alpha_{n}^{*}\right) \text {. }
$$

Proof. Since $\mu_{\alpha_{j}} \leq \mu_{\alpha_{j}^{*}}$ for all $j$, then

$$
\begin{gathered}
\prod_{j=1}^{n}\left(1-\mu_{\alpha_{j}}\right) \frac{T_{j}}{\sum_{j=1}^{n} T_{j}} \geq \prod_{j=1}^{n}\left(1-\mu_{\alpha_{j}^{*}}\right) \frac{T_{j}^{*}}{\sum_{j=1}^{n} T_{j}^{*}} ; \\
1-\prod_{j=1}^{n}\left(1-\mu_{\alpha_{j}}\right) \frac{T_{j}}{\sum_{j=1}^{n} T_{j}} \leq 1-\prod_{j=1}^{n}\left(1-\mu_{\alpha_{j}^{*}}\right) \frac{T_{j}^{*}}{\sum_{j=1}^{n} T_{j}^{*}} .
\end{gathered}
$$

Since $v_{\alpha_{j}} \geq v_{\alpha_{j}^{*}}$, for all $j$, then

$$
\prod_{j=1}^{n}\left(v_{\alpha_{j}}\right)^{\frac{T_{j}}{\sum_{j=1}^{n} T_{j}}} \geq \prod_{j=1}^{n}\left(v_{\alpha_{j}^{*}}\right)^{\frac{T_{j}^{*}}{\sum_{j=1}^{n} T_{j}^{*}}} .
$$


Let $\alpha=\left(\mu_{\alpha}, v_{\alpha}\right)=\operatorname{IFPWA}\left(\alpha_{1}, \alpha_{2}, \ldots, \alpha_{n}\right), \alpha^{*}=\left(\mu_{\alpha^{*}}, v_{\alpha^{*}}\right)=\operatorname{IFPWA}\left(\alpha_{1}^{*}, \alpha_{2}^{*}, \ldots, \alpha_{n}^{*}\right)$, we have

$$
\mu_{\alpha} \leq \mu_{\alpha^{*}}, v_{\alpha} \geq v_{\alpha^{*}}
$$

$$
\operatorname{IFPWA}\left(\alpha_{1}, \alpha_{2}, \ldots, \alpha_{n}\right) \leq_{L} \operatorname{IFPWA}\left(\alpha_{1}^{*}, \alpha_{2}^{*}, \ldots, \alpha_{n}^{*}\right) \text {. }
$$

Based on remark 1, we have,

$$
\operatorname{IFPWA}\left(\alpha_{1}, \alpha_{2}, \ldots, \alpha_{n}\right) \leq \operatorname{IFPWA}\left(\alpha_{1}^{\star}, \alpha_{2}^{*}, \ldots, \alpha_{n}^{*}\right),
$$

which complete the proof of Theorem 4 .

Theorem 5. Let $\alpha_{j}=\left(\mu_{\alpha_{j}}, v_{\alpha_{j}}\right) \quad(j=1,2, \ldots, n)$ be a collections of IFVs, $T_{j}=\prod_{k=1}^{j-1} S\left(\alpha_{k}\right)$ $(j=2, \ldots, n), T_{1}=1$ and $S\left(\alpha_{k}\right)$ is the score of IFV $\alpha_{k}$, if $\beta=\left(\mu_{\beta}, v_{\beta}\right)$ is an intuitionistic fuzzy value on $X$, then

$$
\operatorname{IFPWA}\left(\alpha_{1} \oplus \beta, \alpha_{2} \oplus \beta, \ldots, \alpha_{n} \oplus \beta\right)=\operatorname{IFPWA}\left(\alpha_{1}, \alpha_{2}, \ldots, \alpha_{n}\right) \oplus \beta .
$$

Proof. Since

$$
\alpha_{j} \oplus \beta=\left(\mu_{\alpha_{j}}+\mu_{\beta}-\mu_{\alpha_{j}} \mu_{\beta}, v_{\alpha_{j}} v_{\beta}\right)=\left(1-\left(1-\mu_{\alpha_{j}}\right)\left(1-\mu_{\beta}\right), v_{\alpha_{j}} v_{\beta}\right) \text {. }
$$

According to Theorem 1, we have

$$
\begin{gathered}
\operatorname{IFPWA}\left(\alpha_{1} \oplus \beta, \alpha_{2} \oplus \beta, \ldots, \alpha_{n} \oplus \beta\right)= \\
\left(1-\prod_{j=1}^{n}\left(\left(1-\mu_{\alpha_{j}}\right)\left(1-\mu_{\beta}\right)\right) \frac{T_{j}}{\sum_{j=1}^{n} T_{j}}, \prod_{j=1}^{n}\left(v_{\alpha_{j}} v_{\beta}\right) \frac{T_{j}}{\sum_{j=1}^{n} T_{j}}\right)= \\
\left(1-\left(1-\mu_{\beta}\right)_{j=1}^{n}\left(\frac{T_{j}}{\sum_{j=1}^{n} T_{j}}\right) \prod_{j=1}^{n}\left(1-\mu_{\alpha_{j}}\right) \frac{T_{j}}{\sum_{j=1}^{n} T_{j}},\left(v_{\beta}\right) \sum_{j=1}^{n}\left(\frac{T_{j}}{\sum_{j=1}^{n} T_{j}}\right) \prod_{j=1}^{n}\left(v_{\alpha_{j}}\right) \frac{T_{j}}{\sum_{j=1}^{n} T_{j}}\right)= \\
\left.1-\left(1-\mu_{\beta}\right) \prod_{j=1}^{n}\left(1-\mu_{\alpha_{j}}\right) \frac{T_{j}}{\sum_{j=1}^{n} T_{j}}, v_{\beta} \prod_{j=1}^{n}\left(v_{\alpha_{j}}\right) \frac{T_{j}}{\sum_{j=1}^{n} T_{j}}\right) .
\end{gathered}
$$

According to Definition 3 and the operational laws of IFVs, we have

$$
\begin{gathered}
\operatorname{IFPWA}\left(\alpha_{1}, \alpha_{2}, \ldots, \alpha_{n}\right) \oplus \beta= \\
\left(1-\prod_{j=1}^{n}\left(1-\mu_{\alpha_{j}}\right)^{\frac{T_{j}}{\sum_{j=1}^{n} T_{j}}}, \prod_{j=1}^{n}\left(v_{\alpha_{j}}\right)^{\frac{T_{j}}{\sum_{j=1}^{n} T_{j}}}\right) \oplus\left(\mu_{\beta}, v_{\beta}\right)= \\
\left(1-\left(1-\mu_{\beta}\right) \prod_{j=1}^{n}\left(1-\mu_{\alpha_{j}}\right) \frac{T_{j}}{\sum_{j=1}^{n} T_{j}}, v_{\beta} \prod_{j=1}^{n}\left(v_{\alpha_{j}}\right) \frac{T_{j}}{\sum_{j=1}^{n} T_{j}}\right) .
\end{gathered}
$$


Thus,

$$
\operatorname{IFPWA}\left(\alpha_{1} \oplus \beta, \alpha_{2} \oplus \beta, \ldots, \alpha_{n} \oplus \beta\right)=\operatorname{IFPWA}\left(\alpha_{1}, \alpha_{2}, \ldots, \alpha_{n}\right) \oplus \beta .
$$

Theorem 6. Let $\alpha_{j}=\left(\mu_{\alpha_{j}}, v_{\alpha_{j}}\right) \quad(j=1,2, \ldots, n)$ be a collections of IFVs, $T_{j}=\prod_{k=1}^{j-1} S\left(\alpha_{k}\right)$ $(j=2, \ldots, n), T_{1}=1$ and $S\left(\alpha_{k}\right)$ be the score of IFV $\alpha_{k}$, If $r>0$, then

$$
\operatorname{IFPWA}\left(r \alpha_{1}, r \alpha_{2}, \ldots, r \alpha_{n}\right)=r \operatorname{IFPWA}\left(\alpha_{1}, \alpha_{2}, \ldots, \alpha_{n}\right) .
$$

Proof. According to operation laws described in Section 1, we have

$$
r \alpha_{j}=\left(1-\left(1-\mu_{\alpha_{j}}\right)^{r},\left(v_{\alpha_{j}}\right)^{r}\right) .
$$

According to Theorem 1, we have

$$
\begin{aligned}
& \operatorname{IFPWA}\left(r \alpha_{1}, r \alpha_{2}, \ldots, r \alpha_{n}\right)=\left(1-\prod_{j=1}^{n}\left(\left(1-\mu_{\alpha_{j}}\right)^{r}\right) \frac{T_{j}}{\sum_{j=1}^{n} T_{j}}, \prod_{j=1}^{n}\left(\left(v_{\alpha_{j}}\right)^{r}\right) \frac{T_{j}}{\sum_{j=1}^{n} T_{j}}\right)= \\
& \left(1-\prod_{j=1}^{n}\left(1-\mu_{\alpha_{j}}\right)^{r \frac{T_{j}}{\sum_{j=1}^{n} T_{j}}}, \prod_{j=1}^{n}\left(v_{\alpha_{j}}\right)^{r \frac{T_{j}}{\sum_{j=1}^{n} T_{j}}}\right) \\
& r \operatorname{IFPWA}\left(\alpha_{1}, \alpha_{2}, \ldots, \alpha_{n}\right)=r\left(1-\prod_{j=1}^{n}\left(1-\mu_{\alpha_{j}}\right)^{\frac{T_{j}}{\sum_{j=1}^{n} T_{j}}}, \prod_{j=1}^{n}\left(v_{\alpha_{j}}\right)^{\frac{T_{j}}{\sum_{j=1}^{n} T_{j}}}\right)= \\
& \left(1-\left(\prod_{j=1}^{n}\left(1-\mu_{\alpha_{j}}\right)^{\frac{T_{j}}{\sum_{j=1}^{n} T_{j}}}\right)^{r},\left(\prod_{j=1}^{n}\left(v_{\alpha_{j}}\right)^{\frac{T_{j}}{\sum_{j=1}^{n} T_{j}}}\right)^{r}\right)= \\
& \left(1-\prod_{j=1}^{n}\left(1-\mu_{\alpha_{j}}\right)^{r \frac{T_{j}}{\sum_{j=1}^{n} T_{j}}}, \prod_{j=1}^{n}\left(v_{\alpha_{j}}\right)^{r \frac{T_{j}}{\sum_{j=1}^{n} T_{j}}}\right) \text {. }
\end{aligned}
$$

Thus

$$
\operatorname{IFPWA}\left(r \alpha_{1}, r \alpha_{2}, \ldots, r \alpha_{n}\right)=r \operatorname{IFPWA}\left(\alpha_{1}, \alpha_{2}, \ldots, \alpha_{n}\right) .
$$

According to Theorems 5 and 6 , we can get Theorem 7 easily.

Theorem 7. Let $\alpha_{j}=\left(\mu_{\alpha_{j}}, v_{\alpha_{j}}\right) \quad(j=1,2, \ldots, n)$ be a collections of IFVs, $T_{j}=\prod_{k=1}^{j-1} S\left(\alpha_{k}\right)$ $(j=2, \ldots, n), T_{1}=1$ and $S\left(\alpha_{k}\right)$ is the score of IFV $\alpha_{k}$, If $r>0, \beta=\left(\mu_{\beta}, v_{\beta}\right)$ is an intuitionistic fuzzy value on $X$, then,

$$
\operatorname{IFPWA}\left(r \alpha_{1} \oplus \beta, r \alpha_{2} \oplus \beta, \ldots, r \alpha_{n} \oplus \beta\right)=r \operatorname{IFPWA}\left(\alpha_{1}, \alpha_{2}, \ldots, \alpha_{n}\right) \oplus \beta \text {. }
$$


Theorem 8. Let $\alpha_{j}=\left(\mu_{\alpha_{j}}, v_{\alpha_{j}}\right)$ and $\beta_{j}=\left(\mu_{\beta_{j}}, v_{\beta_{j}}\right) \quad(j=1,2, \ldots, n)$ be two collections of IFVs, $T_{j}=\prod_{k=1}^{j-1} S\left(\alpha_{k}\right)(j=2, \ldots, n), T_{1}=1$ and $S\left(\alpha_{k}\right)$ is the score of IFV $\alpha_{k}$, then, $\operatorname{IFPWA}\left(\alpha_{1} \oplus \beta_{1}, \alpha_{2} \oplus \beta_{2}, \ldots, \alpha_{n} \oplus \beta_{n}\right)=\operatorname{IFPWA}\left(\alpha_{1}, \alpha_{2}, \ldots, \alpha_{n}\right) \oplus \operatorname{IFPWA}\left(\beta_{1}, \beta_{2}, \ldots, \beta_{n}\right)$.

Proof: According to Definition 3, we have

$$
\begin{aligned}
& \operatorname{IFPWA}\left(\alpha_{1} \oplus \beta_{1}, \alpha_{2} \oplus \beta_{2}, \ldots, \alpha_{n} \oplus \beta_{n}\right)= \\
& \left(1-\prod_{j=1}^{n}\left(\left(1-\mu_{\alpha_{j}}\right)\left(1-\mu_{\beta_{j}}\right)\right) \frac{T_{j}}{\sum_{j=1}^{n} T_{j}}, \prod_{j=1}^{n}\left(v_{\alpha_{j}} v_{\beta_{j}}\right)^{\frac{T_{j}}{\sum_{j=1}^{n} T_{j}}}\right)= \\
& \left(1-\prod_{j=1}^{n}\left(1-\mu_{\alpha_{j}}\right)^{\frac{T_{j}}{\sum_{j=1}^{n} T_{j}}} \prod_{j=1}^{n}\left(1-\mu_{\beta_{j}}\right)^{\frac{T_{j}}{\sum_{j=1}^{n} T_{j}}}, \prod_{j=1}^{n}\left(v_{\alpha_{j}}\right)^{\frac{T_{j}}{\sum_{j=1}^{n} T_{j}}} \prod_{j=1}^{n}\left(v_{\beta_{j}}\right)^{\frac{T_{j}}{\sum_{j=1}^{n} T_{j}}}\right) ; \\
& \operatorname{IFPWA}\left(\alpha_{1}, \alpha_{2}, \ldots, \alpha_{n}\right) \oplus \operatorname{IFPWA}\left(\beta_{1}, \beta_{2}, \ldots, \beta_{n}\right)= \\
& \left(1-\prod_{j=1}^{n}\left(1-\mu_{\alpha_{j}}\right)^{\frac{T_{j}}{\sum_{j=1}^{n} T_{j}}}, \prod_{j=1}^{n}\left(v_{\alpha_{j}}\right)^{\frac{T_{j}}{\sum_{j=1}^{n} T_{j}}}\right) \oplus\left(1-\prod_{j=1}^{n}\left(1-\mu_{\beta_{j}}\right)^{\frac{T_{j}}{\sum_{j=1}^{n} T_{j}}}, \prod_{j=1}^{n}\left(v_{\beta_{j}}\right)^{\frac{T_{j}}{\sum_{j=1}^{n} T_{j}}}\right)= \\
& \left(1-\prod_{j=1}^{n}\left(1-\mu_{\alpha_{j}}\right)^{\frac{T_{j}}{\sum_{j=1}^{n} T_{j}}} \prod_{j=1}^{n}\left(1-\mu_{\beta_{j}}\right)^{\frac{T_{j}}{\sum_{j=1}^{n} T_{j}}}, \prod_{j=1}^{n}\left(v_{\alpha_{j}}\right)^{\frac{T_{j}}{\sum_{j=1}^{n} T_{j}}} \prod_{j=1}^{n}\left(v_{\beta_{j}}\right)^{\frac{T_{j}}{\sum_{j=1}^{n} T_{j}}}\right) .
\end{aligned}
$$

Thus,

$\operatorname{IFPWA}\left(\alpha_{1} \oplus \beta_{1}, \alpha_{2} \oplus \beta_{2}, \ldots, \alpha_{n} \oplus \beta_{n}\right)=\operatorname{IFPWA}\left(\alpha_{1}, \alpha_{2}, \ldots, \alpha_{n}\right) \oplus \operatorname{IFPWA}\left(\beta_{1}, \beta_{2}, \ldots, \beta_{n}\right)$.

Based on the IFPWA operator and the geometric mean, here we define an intuitionistic fuzzy prioritized weighted geometric (IFPWG) operator.

Definition 4. Let $\alpha_{j}=\left(\mu_{\alpha_{j}}, v_{\alpha_{j}}\right) \quad(j=1,2, \ldots, n)$ be a collection of IFVs, and let IFPWG: $V^{n} \rightarrow V$, if

$$
\operatorname{IFPWG}_{\lambda}\left(\alpha_{1}, \alpha_{2}, \ldots, \alpha_{n}\right)=\alpha_{1}^{\frac{T_{1}}{\sum_{j=1}^{n} T_{j}}} \otimes \alpha_{2}^{\frac{T_{2}}{\sum_{j=1}^{n} T_{j}}} \otimes \cdots \otimes \alpha_{n} \frac{T_{n}}{\sum_{j=1}^{n} T_{j}},
$$

then the function IFPWG is called an intuitionistic fuzzy prioritized weighted aggregation (IFPWG) operator, where $T_{j}=\prod_{k=1}^{j-1} S\left(\alpha_{k}\right) \quad(j=2, \ldots, n), T_{1}=1$ and $S\left(\alpha_{k}\right)$ is the score of IFV $\alpha_{k}$. 
Theorem 9. Let $\alpha_{j}=\left(\mu_{\alpha_{j}}, v_{\alpha_{j}}\right) \quad(j=1,2, \ldots, n)$ be a collection of IFVs, then their aggregated value by using the IFPWG operator is also an IFV, and

$$
\operatorname{IFPWG}\left(\alpha_{1}, \alpha_{2}, \ldots, \alpha_{n}\right)=\left(\prod_{j=1}^{n}\left(\mu_{\alpha_{j}}\right)^{\frac{T_{j}}{\sum_{j=1}^{n} T_{j}}}, 1-\prod_{j=1}^{n}\left(1-v_{\alpha_{j}}\right)^{\frac{T_{j}}{\sum_{j=1}^{n} T_{j}}}\right),
$$

where $T_{j}=\prod_{k=1}^{j-1} S\left(\alpha_{k}\right) \quad(j=2, \ldots, n), T_{1}=1$ and $S\left(\alpha_{k}\right)$ is the score of IFV $\alpha_{k}$.

Proof: The proof of Theorem 9 is similar to Theorem 1.

Theorem 10. (Idempotency) Let $\alpha_{j}=\left(\mu_{\alpha_{j}}, v_{\alpha_{j}}\right)(j=1,2, \ldots, n)$ be a collection of IFVs, where $T_{j}=\prod_{k=1}^{j-1} S\left(\alpha_{k}\right)(j=2, \ldots, n), T_{1}=1$ and $S\left(\alpha_{k}\right)$ is the score of IFV $\alpha_{k}$. If all $\alpha_{j}(j=1,2, \ldots, n)$ are equal, i.e. $\alpha_{j}=\alpha$, for all $j$, then

$$
\operatorname{IFPWG}\left(\alpha_{1}, \alpha_{2}, \ldots, \alpha_{n}\right)=\alpha .
$$

Proof: The proof of Theorem 10 is similar to Theorem 2.

Theorem 11. (Boundary) Let $\alpha_{j}=\left(\mu_{\alpha_{j}}, v_{\alpha_{j}}\right)(j=1,2, \ldots, n)$ be a collection of IFVs, where $T_{j}=\prod_{k=1}^{j-1} S\left(\alpha_{k}\right) \quad(j=2, \ldots, n), T_{1}=1$ and $S\left(\alpha_{k}\right)$ is the score of IFV $\alpha_{k}$, and let

$$
\alpha^{-}=\left(\min _{j}\left(\mu_{\alpha_{j}}\right), \max _{j}\left(v_{\alpha_{j}}\right)\right), \alpha^{+}=\left(\max _{j}\left(\mu_{\alpha_{j}}\right), \min _{j}\left(v_{\alpha_{j}}\right)\right) \text {. }
$$

Then

$$
\alpha^{-} \leq \operatorname{IFPWG}\left(\alpha_{1}, \alpha_{2}, \ldots, \alpha_{n}\right) \leq \alpha^{+} .
$$

Proof: The proof of Theorem 11 is similar to Theorem 3.

Theorem 12. (Monotonicity) Let $\alpha_{j}=\left(\mu_{\alpha_{j}}, v_{\alpha_{j}}\right)$ and $\alpha_{j}^{*}=\left(\mu_{\alpha_{j}^{*}}, v_{\alpha_{j}^{*}}\right) \quad(j=1,2, \ldots, n)$ be two collections of IFVs, $T_{j}=\prod_{k=1}^{j-1} S\left(\alpha_{k}\right), T_{j}^{*}=\prod_{k=1}^{j-1} S\left(\alpha_{k}^{*}\right) \quad(j=2, \ldots, n), T_{1}=T_{1}^{*}=1, S\left(\alpha_{k}\right)$ is the score of IFV $\alpha_{k}, S\left(\alpha_{k}^{*}\right)$ is the score of IFV $\alpha_{k}^{*}$ if $\mu_{\alpha_{j}} \leq \mu_{\alpha_{j}^{*}}$ and $v_{\alpha_{j}} \geq v_{\alpha_{j}^{*}}$, for all $j$, then

$$
\operatorname{IFPWG}\left(\alpha_{1}, \alpha_{2}, \ldots, \alpha_{n}\right) \leq \operatorname{IFPWG}\left(\alpha_{1}^{\star}, \alpha_{2}^{*}, \ldots, \alpha_{n}^{*}\right) \text {. }
$$

Proof: The proof of Theorem 12 is similar to Theorem 4 .

Theorem 13. Let $\alpha_{j}=\left(\mu_{\alpha_{j}}, v_{\alpha_{j}}\right) \quad(j=1,2, \ldots, n)$ be a collection of IFVs, $T_{j}=\prod_{k=1}^{j-1} S\left(\alpha_{k}\right)$ $(j=2, \ldots, n), T_{1}=1$ and $S\left(\alpha_{k}\right)$ be the score of IFV $\alpha_{k}$, if $\beta=\left(\mu_{\beta}, v_{\beta}\right)$ is an intuitionistic fuzzy value on $X$, then

$$
\operatorname{IFPWG}\left(\alpha_{1} \otimes \beta, \alpha_{2} \otimes \beta, \ldots, \alpha_{n} \otimes \beta\right)=\operatorname{IFPWG}\left(\alpha_{1}, \alpha_{2}, \ldots, \alpha_{n}\right) \otimes \beta .
$$

Proof: The proof of Theorem 13 is similar to Theorem 5. 
Theorem 14. Let $\alpha_{j}=\left(\mu_{\alpha_{j}}, v_{\alpha_{j}}\right) \quad(j=1,2, \ldots, n)$ be a collections of IFVs, $T_{j}=\prod_{k=1}^{j-1} S\left(\alpha_{k}\right)$ $(j=2, \ldots, n), T_{1}=1$ and $S\left(\alpha_{k}\right)$ is the score of IFV $\alpha_{k}$, If $r>0$, then,

$$
\operatorname{IFPWG}\left(\left(\alpha_{1}\right)^{r},\left(\alpha_{2}\right)^{r}, \ldots,\left(\alpha_{n}\right)^{r}\right)=\left(\operatorname{IFPWG}\left(\alpha_{1}, \alpha_{2}, \ldots, \alpha_{n}\right)\right)^{r} .
$$

Proof: The proof of Theorem 14 is similar to Theorem 6.

Similar to Theorem 7, we can drive Theorem 15 easily.

Theorem 15. Let $\alpha_{j}=\left(\mu_{\alpha_{j}}, v_{\alpha_{j}}\right) \quad(j=1,2, \ldots, n)$ be a collections of IFVs, $T_{j}=\prod_{k=1}^{j-1} S\left(\alpha_{k}\right)$ $(j=2, \ldots, n), T_{1}=1$ and $S\left(\alpha_{k}\right)$ is the score of IFV $\alpha_{k}$, If $r>0, \beta=\left(\mu_{\beta}, v_{\beta}\right)$ is an intuitionistic fuzzy value on $X$, then,

$$
\operatorname{IFPWG}\left(\left(\alpha_{1}\right)^{r} \otimes \beta,\left(\alpha_{2}\right)^{r} \otimes \beta, \ldots,\left(\alpha_{n}\right)^{r} \otimes \beta\right)=\left(\operatorname{IFPWG}\left(\alpha_{1}, \alpha_{2}, \ldots, \alpha_{n}\right)\right)^{r} \otimes \beta .
$$

Theorem 16. Let $\alpha_{j}=\left(\mu_{\alpha_{j}}, v_{\alpha_{j}}\right)$ and $\beta_{j}=\left(\mu_{\beta_{j}}, v_{\beta_{j}}\right)(j=1,2, \ldots, n)$ be two collections of IFVs, $T_{j}=\prod_{k=1}^{j-1} S\left(\alpha_{k}\right)(j=2, \ldots, n), T_{1}=1$ and $S\left(\alpha_{k}\right)$ is the score of IFV $\alpha_{k}$, then, $\operatorname{IFPWG}\left(\alpha_{1} \otimes \beta_{1}, \alpha_{2} \otimes \beta_{2}, \ldots, \alpha_{n} \otimes \beta_{n}\right)=\operatorname{IFPWG}\left(\alpha_{1}, \alpha_{2}, \ldots, \alpha_{n}\right) \oplus \operatorname{IFPWG}\left(\beta_{1}, \beta_{2}, \ldots, \beta_{n}\right) .(63)$

Proof: The proof of Theorem 16 is similar to Theorem 8.

\section{An approach to multi-criteria group decision making under intuitionistic fuzzy environment}

In this Section, we utilize the proposed aggregation operators to group decision making under intuitionistic fuzzy environment.

In a group decision making problem, suppose $X=\left\{x_{1}, x_{2}, \cdots, x_{m}\right\}$ is the set of alternatives, Let $C=\left\{C_{1}, C_{2}, \ldots, C_{n}\right\}$ be a collection of criteria and that there is a prioritization between the criteria expressed by the linear ordering $C_{1} \succ C_{2} \succ C_{3} \ldots \succ C_{n}$ indicates criteria $C_{j}$ has a higher priority than $C_{i}$ if $j<i$, and $E=\left\{e_{1}, e_{2}, \cdots, e_{p}\right\}$ is the set of decision makers and that there is a prioritization between the decision makers expressed by the linear ordering $e_{1} \succ e_{2} \ldots \succ e_{p}$ indicate criteria $e_{\varsigma}$ has a higher priority than $e_{\tau}$ if $\varsigma<\tau$. Let $D^{(q)}=\left(d_{i j}^{(q)}\right)_{m \times n}$ be an intuitionistic fuzzy decision matrix, and $d_{i j}^{(q)}=\left(t_{i j}^{(q)}, f_{i j}^{(q)}, \pi_{i j}^{(q)}\right)$ be an attribute value provided by the decision maker $e_{q}$, which is expressed in an IFV. where $t_{i j}^{(q)}$ indicates the degree of the alternative $x_{i}$ satisfies the attribute $C_{j}$ expressed by the decision maker $e_{q}$, $f_{i j}^{(q)}$ indicates the degree of the alternative $x_{i}$ does not satisfy the attribute $C_{j}$ expressed by the decision maker $e_{q}, \pi_{i j}^{(q)}$ indicates the indeterminacy degree corresponding, such that

$$
\begin{gathered}
t_{i j}^{(q)} \in[0,1], f_{i j}^{(q)} \in[0,1], t_{i j}^{(q)}+f_{i j}^{(q)} \leq 1, \pi_{i j}^{(q)}=1-t_{i j}^{(q)}-f_{i j}^{(q)}, \\
i=1,2, \ldots, m ; j=1,2, \ldots, n .
\end{gathered}
$$


If all the attributes $C_{j}(j=1,2, \ldots, n)$ are of the same type, then the attribute values do not need normalization. Whereas, there are generally benefit attributes (the bigger the attribute values the better) and cost attributes (the smaller the attribute values the better) in multi-attribute decision making (Xu, Hu 2010). We may transform the attribute values of cost type into the attribute values of benefit type, in such a case, $D^{(q)}=\left(d_{i j}^{(q)}\right)_{m \times n}$ can be transformed into the intuitionistic fuzzy decision matrices $R^{(q)}=\left(r_{i j}^{(q)}\right)_{m \times n}$, where $r_{i j}^{(q)}=\left(\mu_{i j}^{(q)}, v_{i j}^{(q)}, \pi_{i j}^{(q)}\right)$, and

$$
r_{i j}^{(q)}=\left(\mu_{i j}^{(q)}, v_{i j}^{(q)}, \pi_{i j}^{(q)}\right)=\left\{\begin{array}{l}
d_{i j}^{(q)}, \text { for benefit attribute } C_{j} \\
\bar{d}_{i j}^{(q)}, \text { for cost attribute } C_{j}
\end{array} \quad i=1,2, \ldots, m, j=1,2, \ldots, n,\right.
$$

where $\bar{d}_{i j}^{(q)}$ is the complement of $d_{i j}^{(q)}$ such that

$$
d_{i j}^{(q)}=\left(f_{i j}^{(q)}, t_{i j}^{(q)}, \pi_{i j}^{(q)}\right), \pi_{i j}^{(q)}=1-t_{i j}^{(q)}-f_{i j}^{(q)}=1-\mu_{i j}^{(q)}-v_{i j}^{(q)}, i=1,2, \ldots, m ; j=1,2, \ldots, n .
$$

Then, we utilize the IFPWA (or IFPWG) operator to develop an approach to multi-criteria decision making under intuitionistic fuzzy environment, the main steps are as follows:

Step 1. Calculate the values of $T_{i j}^{(q)},(q=1,2, \ldots, p)$ based on the following equations.

$$
\begin{gathered}
T_{i j}^{(q)}=\prod_{k=1}^{q-1} S\left(r_{i j}^{(q)}\right)(q=2, \ldots, p) ; \\
T_{i j}^{(1)}=1 .
\end{gathered}
$$

Step 2. Utilize the IFPWA operator

$$
r_{i j}=\operatorname{IFPWA}\left(r_{i j}^{(1)}, r_{i j}^{(2)}, \ldots, r_{i j}^{(p)}\right)=\left(1-\prod_{q=1}^{p}\left(1-\mu_{i j}^{(q)}\right)^{\frac{T_{i j}^{(q)}}{\sum_{q=1}^{p} T_{i j}^{(q)}}}, \prod_{q=1}^{p}\left(v_{i j}^{(q)}\right)^{\frac{T_{i j}^{(q)}}{\sum_{q=1}^{p} T_{i j}^{(q)}}}\right),
$$

or the IFPWG operator:

$$
r_{i j}=\operatorname{IFPWG}\left(r_{i j}^{(1)}, r_{i j}^{(2)}, \ldots, r_{i j}^{(p)}\right)=\left(\prod_{q=1}^{p}\left(\mu_{i j}^{(q)}\right)^{\frac{T_{i j}^{(q)}}{\sum_{q=1}^{p} T_{i j}^{(q)}}}, 1-\prod_{q=1}^{p}\left(1-v_{i j}^{(q)}\right)^{\frac{T_{i j}^{(q)}}{\sum_{q=1}^{p} T_{i j}^{(q)}}}\right) .
$$

To aggregate all the individual intuitionistic fuzzy decision matrix $R^{(q)}=\left(r_{i j}^{(q)}\right)_{m \times n}(q=1,2, \ldots, p)$ into the collective intuitionistic fuzzy decision matrix $R=\left(r_{i j}\right)_{m \times n}, i=1,2, \ldots, m ; j=1,2, \ldots, n$.

Step 3. Calculate the values of $T_{i j},(i=1,2, \ldots, m, j=1,2, \ldots, n)$ based on following equations.

$$
\begin{gathered}
T_{i j}=\prod_{k=1}^{j-1} S\left(r_{i k}\right)(i=1,2, \ldots, m, j=2, \ldots, p) ; \\
T_{i 1}=1 \quad i=1,2, \ldots, m .
\end{gathered}
$$


Step 4. Aggregate the intuitionistic fuzzy values $r_{i j}$ for each alternative $x_{i}$ by the IFPWA (or IFPWG) operator:

$$
r_{i}=\operatorname{IFPWA}\left(r_{i 1}, r_{i 2}, \ldots, r_{i n}\right)=\left(1-\prod_{j=1}^{n}\left(1-\mu_{i j}\right)^{\frac{T_{i j}}{\sum_{j=1}^{n} T_{i j}}}, \prod_{j=1}^{n}\left(v_{i j}\right)^{\frac{T_{i j}}{\sum_{j=1}^{n} T_{i j}}}\right) \quad i=1,2, \ldots, m,
$$

or

$$
r_{i}=\operatorname{IFPWG}\left(r_{i 1}, r_{i 2}, \ldots, r_{i n}\right)=\left(\prod_{j=1}^{n}\left(\mu_{i j}\right)^{\frac{T_{i j}}{\sum_{j=1}^{n} T_{i j}}}, 1-\prod_{j=1}^{n}\left(1-v_{i j}\right)^{\frac{T_{i j}}{\sum_{j=1}^{n} T_{i j}}}\right) \quad i=1,2, \ldots, m .
$$

Step 5. Rank all the alternatives by the score function described in Section 1

$$
S\left(r_{i}\right)=\frac{1+\mu_{r_{i}}-v_{r_{i}}}{2}, i=1,2, \cdots, m,
$$

then the bigger the value of $s\left(r_{i}\right)$, the larger the overall IFV $r_{i}$ and thus the alternative $x_{i}(i=1,2, \ldots, m)$.

\section{Practical example}

Work to strengthen academic education, promote the building of teaching body, the school of management in a Chinese university wants to introduce oversea outstanding teachers. This introduction has raised great attention from the school; university president $e_{1}$, dean of management school $e_{2}$, and human resource officer $e_{3}$ sets up the panel of decision makers which will take the whole responsibility for this introduction. They made strict evaluation for 5 candidates $x_{i}(i=1,2, \ldots, 5)$ from four aspects, namely morality $C_{1}$ research capability $C_{2}$ teaching skill $C_{3}$, educational background $C_{4}$. University president have the absolute priority for decision making, dean of the management school comes next. Besides, this introduction will be in strict accordance with the principle of combined ability with political integrity. The prioritization relationship for the criteria is as below, $C_{1} \succ C_{2} \succ C_{3} \succ C_{4}$. Three decision makers evaluated the candidates $x_{i}(i=1,2,3,4,5)$ with respect to the attributes $C_{j}(j=1,2, \ldots, 4)$ and construct the following three intuitionistic fuzzy decision $D^{(q)}=\left(d_{i j}^{(q)}\right)_{5 \times 4} \quad(q=1,2,3)$ (see Tables 1-3). Since all the attributes $C_{j}(j=1,2, \ldots, 5)$ are of the benefit type, then the attribute values do not need normalization, therefore, $R^{(q)}=D^{(q)}=\left(d_{i j}^{(q)}\right)_{5 \times 4}=\left(r_{i j}^{(q)}\right)_{5 \times 4}$.

Table 1. Intuitionistic fuzzy decision matrix $R^{(1)}$

\begin{tabular}{ccccc}
\hline & $C_{1}$ & $C_{2}$ & $C_{3}$ & $C_{4}$ \\
\hline$x_{1}$ & $(0.9,0.0)$ & $(0.6,0.3)$ & $(0.75,0.15)$ & $(0.9,0.0)$ \\
$x_{2}$ & $(0.9,0.0)$ & $(0.75,0.15)$ & $(0.75,0.15)$ & $(0.75,0.15)$ \\
$x_{3}$ & $(0.9,0.0)$ & $(0.75,0.15)$ & $(0.75,0.15)$ & $(0.45,0.45)$ \\
$x_{4}$ & $(0.75,0.15)$ & $(0.75,0.15)$ & $(0.9,0.0)$ & $(0.3,0.6)$ \\
$x_{5}$ & $(0.75,0.15)$ & $(0.6,0.3)$ & $(0.75,0.15)$ & $(0.6,0.3)$ \\
\hline
\end{tabular}


Table 2. Intuitionistic fuzzy decision matrix $R^{(2)}$

\begin{tabular}{ccccc}
\hline & $C_{1}$ & $C_{2}$ & $C_{3}$ & $C_{4}$ \\
\hline$x_{1}$ & $(0.75,0.15)$ & $(0.75,0.15)$ & $(0.9,0.0)$ & $(0.3,0.6)$ \\
$x_{2}$ & $(0.75,0.15)$ & $(0.9,0.0)$ & $(0.75,0.15)$ & $(0.75,0.15)$ \\
$x_{3}$ & $(0.9,0.0)$ & $(0.9,0.0)$ & $(0.75,0.15)$ & $(0.6,0.3)$ \\
$x_{4}$ & $(0.9,0.0)$ & $(0.3,0.6)$ & $(0.75,0.15)$ & $(0.6,0.3)$ \\
$x_{5}$ & $(0.45,0.45)$ & $(0.6,0.3)$ & $(0.9,0.0)$ & $(0.9,0.0)$ \\
\hline
\end{tabular}

Table 3. Intuitionistic fuzzy decision matrix $R^{(3)}$

\begin{tabular}{ccccc}
\hline & $C_{1}$ & $C_{2}$ & $C_{3}$ & $C_{4}$ \\
\hline$x_{1}$ & $(0.75,0.15)$ & $(0.9,0.0)$ & $(0.75,0.15)$ & $(0.3,0.6)$ \\
$x_{2}$ & $(0.6,0.3)$ & $(0.75,0.15)$ & $(0.9,0.0)$ & $(0.6,0.3)$ \\
$x_{3}$ & $(0.9,0.0)$ & $(0.6,0.3)$ & $(0.75,0.15)$ & $(0.9,0.0)$ \\
$x_{4}$ & $(0.9,0.0)$ & $(0.75,0.15)$ & $(0.75,0.15)$ & $(0.75,0.15)$ \\
$x_{5}$ & $(0.75,0.15)$ & $(0.75,0.15)$ & $(0.9,0.0)$ & $(0.45,0.45)$ \\
\hline
\end{tabular}

Based on the IFPWA operator, the main steps are as follows.

Step 1. Calculate the values of $T_{i j}^{(1)}, T_{i j}^{(2)}, T_{i j}^{(3)}$ based on Eqs. (66) and (67).

$$
\begin{aligned}
T_{i j}^{(1)}= & \left(\begin{array}{llll}
1 & 1 & 1 & 1 \\
1 & 1 & 1 & 1 \\
1 & 1 & 1 & 1 \\
1 & 1 & 1 & 1 \\
1 & 1 & 1 & 1
\end{array}\right), \quad T_{i j}^{(2)}=\left(\begin{array}{llll}
0.95 & 0.65 & 0.8 & 0.95 \\
0.95 & 0.8 & 0.8 & 0.8 \\
0.95 & 0.8 & 0.8 & 0.5 \\
0.8 & 0.8 & 0.95 & 0.35 \\
0.8 & 0.65 & 0.8 & 0.65
\end{array}\right), \\
T_{i j}^{(3)} & =\left(\begin{array}{llll}
0.76 & 0.52 & 0.76 & 0.3325 \\
0.76 & 0.76 & 0.64 & 0.64 \\
0.9025 & 0.76 & 0.64 & 0.325 \\
0.76 & 0.28 & 0.76 & 0.2275 \\
0.4 & 0.4225 & 0.76 & 0.6175
\end{array}\right)
\end{aligned}
$$

Step 2. Utilize the IFPWA operator (Eq. (68)) to aggregate all the individual intuitionistic fuzzy decision matrix $R^{(q)}=\left(r_{i j}^{(q)}\right)_{5 \times 4} \quad(q=1,2,3)$ into the collective intuitionistic fuzzy decision matrix $R=\left(r_{i j}\right)_{5 \times 4}$ (see table 4 ).

Step 3. Calculate the values of $T_{i j},(i=1,2, \ldots, m, j=1,2, \ldots, n)$ based on Eqs. (70) and (71).

$$
T_{i j}=\left(\begin{array}{cccc}
1 & 0.9109 & 0.7973 & 0.7225 \\
1 & 0.8983 & 0.8140 & 0.7340 \\
1 & 0.9500 & 0.8475 & 0.6780 \\
1 & 0.9285 & 0.6373 & 0.5805 \\
1 & 0.7217 & 0.4965 & 0.4610
\end{array}\right) .
$$


Table 4. Intuitionistic fuzzy decision matrix $R$

\begin{tabular}{ccccc}
\hline & $C_{1}$ & $C_{2}$ & $C_{3}$ & $C_{4}$ \\
\hline$x_{1}$ & $(0.8217,0.0000)$ & $(0.7507,0.0000)$ & $(0.8122,0.0000)$ & $(0.7016,0.0000)$ \\
$x_{2}$ & $(0.7966,0.0000)$ & $(0.8122,0.0000)$ & $(0.8034,0.0000)$ & $(0.7172,0.1799)$ \\
$x_{3}$ & $(0.9000,0.0000)$ & $(0.7841,0.0000)$ & $(0.7500,0.1500)$ & $(0.6279,0.0000)$ \\
$x_{4}$ & $(0.8570,0.0000)$ & $(0.6285,0.2557)$ & $(0.8217,0.0000)$ & $(0.4670,0.4212)$ \\
$x_{5}$ & $(0.6670,0.2237)$ & $(0.6365,0.2605)$ & $(0.8570,0.0000)$ & $(0.7068,0.0000)$ \\
\hline
\end{tabular}

Step 4. Utilize the IFPWA operator (Eq. (72)) to aggregate all the preference values $r_{i j}$ $(i=1,2,3,4,5)$ in the $i$ th line of $R$, and get the overall preference values $r_{i}$ :

$$
\begin{gathered}
r_{1}=(0.7802,0.0000), r_{2}=(0.7880,0.0000) \\
r_{3}=(0.8006,0.0000), r_{4}=(0.7473,0.0000), r_{5}=(0.7148,0.0000) .
\end{gathered}
$$

Step 5. Calculate the scores of $r_{i}(i=1,2,3,4,5)$ respectively:

$$
S_{1}=0.8901, S_{2}=0.8940, S_{3}=0.9003, S_{4}=0.8737, S_{5}=0.8574
$$

Since

$$
\mathrm{S}_{3}>\mathrm{S}_{2}>\mathrm{S}_{1}>\mathrm{S}_{4}>\mathrm{S}_{5}
$$

we have

$$
x_{3} \succ x_{2} \succ x_{1} \succ x_{4} \succ x_{5}
$$

Based on the IFPWG operator, the main steps are as follows:

Step $1^{\prime}$. See step 1 .

Step $2^{\prime}$. Utilize the IFPWG operator (Eq. (69)) to aggregate all the individual intuitionistic fuzzy decision matrix $R^{(q)}=\left(r_{i j}^{(q)}\right)_{5 \times 4}(q=1,2,3)$ into the collective intuitionistic fuzzy decision matrix $R^{\prime}=\left(r_{i j}^{\prime}\right)_{5 \times 4}$ (see table 5$)$.

Table 5. Intuitionistic fuzzy decision matrix $R^{\prime}$

\begin{tabular}{ccccc}
\hline & $C_{1}$ & $C_{2}$ & $C_{3}$ & $C_{4}$ \\
\hline$x_{1}$ & $(0.8022,0.0975)$ & $(0.7069,0.1919)$ & $(0.7940,0.1057)$ & $(0.4855,0.4024)$ \\
$x_{2}$ & $(0.7535,0.1453)$ & $(0.7940,0.1057)$ & $(0.7867,0.1130)$ & $(0.7074,0.1922)$ \\
$x_{3}$ & $(0.9000,0.0000)$ & $(0.7431,0.1558)$ & $(0.7500,0.1500)$ & $(0.5509,0.3464)$ \\
$x_{4}$ & $(0.8381,0.0615)$ & $(0.5272,0.3639)$ & $(0.8022,0.0975)$ & $(0.3993,0.4951)$ \\
$x_{5}$ & $(0.6229,0.2744)$ & $(0.6279,0.2727)$ & $(0.8381,0.0615)$ & $(0.6232,0.2739)$ \\
\hline
\end{tabular}


Step 3': Calculate the values of $T_{i j}^{\prime},(i=1,2, \ldots, m, j=1,2, \ldots, n)$ based on Eqs. (70) and (71).

$$
T_{i j}^{\prime}=\left(\begin{array}{cccc}
1 & 0.8524 & 0.6457 & 0.5450 \\
1 & 0.8041 & 0.6788 & 0.5681 \\
1 & 0.9500 & 0.7540 & 0.6032 \\
1 & 0.8883 & 0.5167 & 0.4404 \\
1 & 0.6742 & 0.4572 & 0.4061
\end{array}\right) .
$$

Step $4^{\prime}$ : Utilize the IFPWG operator (Eq. (73)) to aggregate all the preference values $r_{i j}^{\prime}$ $(i=1,2,3,4,5)$ in the $i$ th line of $R^{\prime}$, and get the overall preference values $r_{i}^{\prime}$ :

$$
\begin{gathered}
r_{1}^{\prime}=(0.7061,0.1888), r_{2}^{\prime}=(0.7623,0.1370), \\
r_{3}^{\prime}=(0.7471,0.1506), r_{4}^{\prime}=(0.6415,0.2502), r_{5}^{\prime}=(0.6585,0.2392) .
\end{gathered}
$$

Step 5': Calculate the scores of $r_{i}^{\prime} \quad(i=1,2,3,4,5)$, respectively:

$$
S_{1}^{\prime}=0.7586, S_{2}^{\prime}=0.8127, S_{3}^{\prime}=0.7983, S_{4}^{\prime}=0.6956, S_{5}^{\prime}=0.7097 \text {. }
$$

Since

$$
\mathrm{S}_{2}^{\prime}>S_{3}^{\prime}>S_{1}^{\prime}>S_{5}^{\prime}>S_{4}^{\prime}
$$

we have

$$
x_{2} \succ x_{3} \succ x_{1} \succ x_{5} \succ x_{4}
$$

The optimal decision have changed the sort result by the IFPWG operator is different from that by the IFPWA operator. The IFPWA operator focuses on the impact of overall data while the IFPWG operator highlights the role of individual data. For example when we sort by IFPWG operator several smaller attribute values correlated with candidate $x_{5}$, such as $r_{34}^{(1)}=(0.45,0.45)$ have bigger impact on the variation of its position in the sort, which leads to candidate $x_{3}$ being in the second place.

If the criteria or the decision makers are at the same priority level, then the proposed operators are reduced to the traditional intuitionistic fuzzy aggregation operators. However, there are different priority levels among these four criteria and three decision makers. For example, the candidate is very hard to be selected when he received poor evaluation from the president of the university. From another point of view, if a candidate owns bad moral character, then he is impossible to be selected no matter how good performance he has received on research capabilities, teaching skill and education background. Therefore, we must consider the prioritization among the criteria or the decision makers. To deal with such situations, the IFPWA and the IFPWG operators are useful tools. From the above analysis, the main advantages over the traditional intuitionistic fuzzy operators are not only due to the fact that our operators accommodate the intuitionistic fuzzy environment but also due to the consideration of the prioritization among the criteria and the decision makers, which makes it more feasible and practical. 


\section{Conclusions}

In this paper, we have studied the intuitionistic fuzzy information aggregation problems where there is a prioritization relationship over the criteria, and have proposed the intuitionistic fuzzy prioritized weighted average operator and the intuitionistic fuzzy prioritized weighted geometric operator on the basis of the idea of prioritized average. The significant feature of the proposed operators is that they consider prioritization among the criteria. Some of their desirable properties are investigated in detail. Then, we have applied our operators to develop a method of multi-criteria group decision making under intuitionistic fuzzy environment. Finally, an example is given to illustrate the given method. The proposed multi-criteria group decision making method considers prioritization relationship among these criteria and decision makers, which allows our method to have wider practical application potentials. It is worth noting that the results of this paper can be extended to the interval-valued intuitionistic fuzzy environment.

\section{References}

Atanasov, K. T. 1986. Intuitionistic fuzzy sets, Fuzzy Sets and Systems 20(1): 87-96. http://dx.doi.org/10.1016/S0165-0114(86)80034-3

Beliakov, G; Bustince, H; Goswami, D. P.; Mukherjee, U. K.; Pal, N. R. 2011. On averaging operators for Atanassov's intuitionistic fuzzy sets, Information Sciences 181(6): 1116-1124. http://dx.doi.org/10.1016/j.ins.2010.11.024

Chen, S. M.; Tan, J. M. 1994. Handling multi-criteria fuzzy decision making problems based on vague set theory, Fuzzy Sets and Systems 67(2): 163-172. http://dx.doi.org/10.1016/0165-0114(94)90084-1

Chen, T. Y. 2011. A comparative analysis of score functions for multiple criteria decision making in intuitionistic fuzzy settings, Information Sciences 181(17): 3652-3676.

http://dx.doi.org/10.1016/j.ins.2011.04.030

Deschrijver, G.; Kerre, E. E. 2003. On the relationship between some extensions of fuzzy set theory, Fuzzy Sets and Systems 133(2): 227-235. http://dx.doi.org/10.1016/S0165-0114(02)00127-6

Hong, D. H.; Choi, C. H. 2000. Multi-criteria fuzzy decision-making problems based on vague set theory, Fuzzy Sets and Systems 114(1): 103-113. http://dx.doi.org/10.1016/S0165-0114(98)00271-1

Hung, W. L.; Yang, M. S. 2007. Similarity measures of intuitionistic fuzzy sets based on Lqmetric, International Journal of Approximate Reasoning 46(1): 120-136. http://dx.doi.org/10.1016/j.ijar.2006.10.002

Hung, W. L.; Yang, M. S. 2004. Similarity measures of intuitionistic fuzzy sets based on Hausdorff distance, Pattern Recognition Letters 25(14): 1603-1611. http://dx.doi.org/10.1016/j.patrec.2004.06.006

Li, D. F. 2010. A ratio ranking method of triangular intuitionistic fuzzy numbers and its application to MADM problems, Computers and Mathematics with Applications 60(6): 1557-1570. http://dx.doi.org/10.1016/j.camwa.2010.06.039

Liang, Z. Z.; Shi, P. F. 2003. Similarity measures on intuitionistic fuzzy sets, Pattern Recognition Letters 24(15): 2687-2693. http://dx.doi.org/10.1016/S0167-8655(03)00111-9

Liu, H. W. 2005. Multi-criteria decision making and reasoning methods in an intuitionistic fuzzy or interval-valued fuzzy environment: $\mathrm{PhD}$ Thesis. Shandong University, Jinan, China.

Liu, H. W.; Wang, G. J. 2007. Multi-criteria decision-making methods based on intuitionistic fuzzy sets, European Journal of Operational Research 179(1):220-233. http://dx.doi.org/10.1016/j.ejor.2006.04.009 
Liu, P. D. 2009. Multi-attribute decision-making method research based on interval vague set and TOPSIS method, Technological and Economic Development of Economy 15(3): 453-463. http://dx.doi.org/10.3846/1392-8619.2009.15.453-463

Tan, C. Q.; Chen, X. H. 2010. Intuitionistic fuzzy Choquet integral operator for multi-criteria decision making, Expert Systems with Applications 37(1): 149-157. http://dx.doi.org/10.1016/j.eswa.2009.05.005

Tan, C. Q. 2011. Generalized intuitionistic fuzzy geometric aggregation operator and its application to multi-criteria group decision making, Soft Computing 15(5): 867-876. http://dx.doi.org/10.1007/s00500-010-0554-6

Wei, G. W. 2010a. GRA method for multiple attribute decision making with incomplete weight information in intuitionistic fuzzy setting, Knowledge-based Systems 23(3): 243-247. http://dx.doi.org/10.1016/j.knosys.2010.01.003

Wei, G. W. 2010b. Some induced geometric aggregation operators with intuitionistic fuzzy information and their application to group decision making, Applied Soft Computing 10(2): 423-431. http://dx.doi.org/10.1016/j.asoc.2009.08.009

Wei, G. W. 2011. Gray relational analysis method for intuitionistic fuzzy multiple attribute decision making, Expert Systems with Applications 38(9): 11671-11677. http://dx.doi.org/10.1016/j.eswa.2011.03.048

Wei, G. W.; Wang, H. J.; Lin, R. 2011a. Application of correlation coefficient to interval-valued intuitionistic fuzzy multiple attribute decision making with incomplete weight information, Knowledge and Information Systems 26(2): 337-349. http://dx.doi.org/10.1007/s10115-009-0276-1

Wei, G. W.; Wang, H. J.; Lin, R.; Zhao, X. F. 2011b. Grey relational analysis method for intuitionistic fuzzy multiple attribute decision making with preference information on alternatives, International Journal of Computational Intelligence Systems 4(2): 164-173. http://dx.doi.org/10.2991/ijcis.2011.4.2.5

Wei, G. W.; Zhao, X. F.; Wang, H. J. 2012. An approach to multiple attribute group decision making with interval intuitionistic trapezoidal fuzzy information, Technological and Economic Development of Economy 18(2): 317-330. http://dx.doi.org/10.3846/20294913.2012.676995

Wei, G. W.; Zhao, X. F. 2012. Some induced correlated aggregating operators with intuitionistic fuzzy information and their application to multiple attribute group decision making, Expert Systems with Applications 39(2): 2026-2034. http://dx.doi.org/10.1016/j.eswa.2011.08.031

Xia, M. M.; Xu, Z. S.; Liao, H. C. 2012. Preference relations based on intuitionistic multiplicative information, IEEE Transaction on Fuzzy System 21(1): 113-133. http://dx.doi.org/10.1109/TFUZZ.2012.2202907

Xu, Z. S. 2007. Intuitionistic fuzzy aggregation operators, IEEE Transactions on Fuzzy Systems 15(6): 1179-1187. http://dx.doi.org/10.1109/TFUZZ.2006.890678

Xu, Z. S. 2010. Choquet integrals of weighted intuitionistic fuzzy information, Information Sciences 180(5): 726-736. http://dx.doi.org/10.1016/j.ins.2009.11.011

$\mathrm{Xu}, \mathrm{Z}$. S. 2011a. Approaches to multiple attribute group decision making based on intuitionistic fuzzy power aggregation operators, Knowledge-Based Systems 24(6): 749-760. http://dx.doi.org/10.1016/j.knosys.2011.01.011

Xu, Z. S. 2011b. Intuitionistic fuzzy Bonferroni means, IEEE Transactions on Systems, Man and Cybernetics 41(2): 568-578. http://dx.doi.org/10.1109/TSMCB.2010.2072918

Xu, Z. S; Yager, R. R. 2006. Some geometric aggregation operators based on intuitionistic fuzzy sets, International Journal of General Systems 35(4): 417-433. http://dx.doi.org/10.1080/03081070600574353

Xu, Z. S.; Hu, H. 2010. Projection models for intuitionistic fuzzy multiple attribute decision making, International Journal of Information Technology and Decision Making 9(2): 267-280. http://dx.doi.org/10.1142/S0219622010003816 
Yager, R. R. 2004b. Choquet aggregation using order inducing variables, International Journal of Uncertainty, Fuzziness and Knowledge-Based Systems 12(1): 69-88. http://dx.doi.org/10.1142/S0218488504002667

Yager, R. R. 2004a. Generalized OWA aggregation operators, Fuzzy Optimization and Decision Making 3(1): 93-107. http://dx.doi.org/10.1023/B:FODM.0000013074.68765.97

Yager, R. R.; Gumrahb, G.; Reformatb, M. Z. 2011. Using a web personal evaluation tool - PET for lexicographic multi-criteria service selection, Knowledge-Based Systems 24(7): 929-942. http://dx.doi.org/10.1016/j.knosys.2011.02.004

Yager, R. R. 2009a. On generalized Bonferroni mean operators for multi-criteria aggregation, International Journal of Approximate Reasoning 50(8): 1279-1286. http://dx.doi.org/10.1016/j.ijar.2009.06.004

Yager, R. R.1988. On ordered weighted averaging aggregation operators in multi-criteria decision making, IEEE Transactions on Systems, Man, and Cybernetics 18(1): 183-190. http://dx.doi.org/10.1109/21.87068

Yager, R. R. 2008. Prioritized aggregation operators, International Journal of Approximate Reasoning 48(1): 263-274. http://dx.doi.org/10.1016/j.ijar.2007.08.009

Yager, R. R. 2009b. Prioritized OWA aggregation, Fuzzy Optimization Decision Making 8(3): 245-262. http://dx.doi.org/10.1007/s10700-009-9063-4

Yager, R. R. 2001. The power average operator, IEEE Transactions on Systems, Man and Cybernetics 31(6): 724-731. http://dx.doi.org/10.1109/3468.983429

Yu, D. J. 2012. Group decision making based on generalized intuitionistic fuzzy prioritized geometric operator, International Journal of Intelligent Systems 27(7): 635-661.

Yu, D. J. 2013. Intuitionistic fuzzy geometric Heronian mean aggregation operators, Applied Soft Computing 13(2): 1235-1246. http://dx.doi.org/10.1016/j.asoc.2012.09.021

Yu, D. J.; Wu, Y. Y.; Lu, T. 2012. Interval-valued intuitionistic fuzzy prioritized operators and their application in multi-criteria group decision making, Knowledge-Based Systems 30(6): 57-66. http://dx.doi.org/10.1016/j.knosys.2011.11.004

Zadeh, L. A. 1965. Fuzzy sets, Information and Control 8: 338-353. http://dx.doi.org/10.1016/S0019-9958(65)90241-X

Zhao, H; Xu, Z. S.; Ni, M. F.; Liu. S. S. 2010. Generalized aggregation operators for intuitionistic fuzzy sets, International Journal of Intelligent Systems 25(1): 1-30. http://dx.doi.org/10.1002/int.20386

Dejian YU received the BSc degree in information management and information system from Heilongjiang institute of science and technology, Harbin, China, the MSc degree in System Engineering from Nanjing university of aeronautics and astronautics, Nanjing, China, and the PhD degree in management science and engineering from Southeast University, Nanjing, China, in 2012. He is currently a Lecturer with the School of Information, Zhejiang University of Finance and Economics, Hangzhou, China. His current research interests include aggregation operators, information fusion, and multi-criteria decision making. 